\title{
Can Dynamic Contrast-enhanced MRI Contribute to Improved Assessment of Rectosigmoid Involvement in Deep Infiltrating Endometriosis?
}

\author{
DANIEL HAUSMANN ${ }^{1,2 *}$, VASILIKI PERIGNON ${ }^{1 *}$, REGULA GRABHERR $^{3}$, \\ ELISABETH WEILAND ${ }^{4}$, MARCEL DOMINIK NICKEL $^{4}$, MAURUS MURER $^{5}$, \\ LARS BOSSHARD ${ }^{6}$, MICHAEL PRUMMER ${ }^{6}$ and RAHEL A. KUBIK-HUCH ${ }^{1}$ \\ ${ }^{1}$ Department of Radiology, Kantonsspital Baden, Baden, Switzerland; \\ ${ }^{2}$ Department of Clinical Radiology and Nuclear Medicine, University Medical Center Mannheim, \\ Medical Faculty Mannheim, Heidelberg University, Mannheim, Germany; \\ ${ }^{3}$ Department of Gynecology, Kantonsspital Baden, Baden, Switzerland; \\ ${ }^{4}$ MR Applications Predevelopment, Siemens Healthcare GmbH, Erlangen, Germany; \\ ${ }^{5}$ Department of Pathology, Kantonsspital Baden, Baden, Switzerland; \\ ${ }^{6}$ Nexus Personalized Health Technologies, ETH Zurich, \\ and Swiss Institute for Bioinformatics (SIB), Zurich, Switzerland
}

\begin{abstract}
Background/Aim: To determine whether a prototypical compressed-sensing volume-interpolated breathhold (csVIBE) provides diagnostic value in detecting rectosigmoid infiltration in deep infiltrating endometriosis (DIE). Patients and Methods: csVIBE was employed in 151 women undergoing pelvic magnetic resonance imaging, of whom 43 had undergone surgery for suspected endometriosis. The accuracy of T2-weighted BLADE and BLADE/csVIBE, additional diagnostic value of csVIBE, and diagnostic confidence were rated by two readers. Additionally, the presence of the "mushroom cap sign" was assessed on BLADE and csVIBE. Results: The diagnostic accuracy, sensitivity, and specificity of BLADE and BLADE/csVIBE were not significantly different between Readers $A$ and $B$. For both readers, the confidence in the diagnosis increased with csVIBE, but this increase in the odds ratio was not significant for both readers. Both readers preferred csVIBE over BLADE with regard to detection of the "mushroom cap sign." Conclusion: csVIBE
\end{abstract}

This article is freely accessible online.

*These Authors contributed equally to this study.

ORCID R. A. Kubik-Huch: http://orcid.org/0000-0002-3636-8697

Correspondence to: Professor Daniel Hausmann, MD, Institute of Radiology, Kantonsspital Baden, Im Ergel 1, 5404 Baden, Switzerland. Tel: +41 564863822, e-mail: daniel.hausmann@ksb.ch

Key Words: Endometriosis, deep infiltrating endometriosis, mushroom cap sign, dynamic contrast-enhanced MRI, compressed-sensing. may provide a diagnostic benefit for surgical strategy selection through better delineation of the "mushroom cap sign."

Endometriosis is a common gynecological disease affecting $10 \%$ to $15 \%$ of all women of reproductive age (1). In women with endometriosis, functioning ectopic glands and stroma are found outside the uterus and can invade organs such as the rectosigmoid (2). Deep infiltrating endometriosis (DIE) is defined by the presence of one or more endometriotic nodules deeper than $5 \mathrm{~mm}$ under the peritoneal surface (3, 4). DIE is often accompanied by fibrosis, and the tissue is well innervated. Affected patients may exhibit a wide range of symptoms including severe chronic pain, dyspareunia, and infertility $(5,6)$. Moreover, DIE is associated with an increased risk of ovarian cancer. Approximately $70 \%$ of women with chronic pelvic pain are affected by DIE (7). It is therefore extremely important to continuously improve both diagnostics and therapy.

DIE of the posterior compartment accounts for approximately $90 \%$ of cases in which endometriosis is clinically suspected. Intestinal endometriosis is a serious form of endometriosis with a high prevalence (8-12), and the rectosigmoid is involved in about $90 \%$ of cases of bowel infiltration (13). Symptoms include diarrhea, constipation, tenesmus, and rectal bleeding.

For the initial assessment, transvaginal ultrasound is the imaging method of choice. In complex cases, especially in DIE, magnetic resonance imaging (MRI) is being increasingly used; it is considered a procedure with very high diagnostic accuracy that can be of significant importance for 
surgical planning $(9,10,14-16)$. MRI is also recommended by the European Society of Urogenital Radiology in symptomatic patients with negative ultrasound findings (5). However, laparoscopy with subsequent histopathological detection of endometrial glands or stroma is still considered the gold standard for evaluation (17).

The most important sequence for the detection of pelvic endometriosis is T2-weighted (T2W) imaging without fat suppression (9). Conversely, T1-weighted (T1W) sequences with Dixon fat suppression without contrast agent are best suited for the detection of small endometrial hemorrhagic implants $(18,19)$.

Contrast-enhanced imaging is recommended to differentiate ovarian endometrioma from other indeterminate adnexal lesions and to rule out malignancy (20). However, only a few studies have addressed the value of gadoliniumbased contrast agent administration in the assessment of endometriosis (21-23). Scardapane et al. (22) found that colorectal infiltration can be detected with high accuracy and good interobserver agreement using contrast-enhanced sequences. In contrast, Bazot et al. (23) found no significant benefit of postcontrast sequences over native MRI for the detection of DIE in a retrospective analysis of 158 patients.

In the present study, we compared the performance of $\mathrm{T} 2 \mathrm{~W}$ BLADE versus the combination of T2W BLADE and a prototypical contrast-enhanced, dynamic, compressed-sensingaccelerated volume-interpolated breath-hold examination (csVIBE) with flexible temporal resolution for the detection of rectal infiltration in DIE. The image quality of this innovative sequence has already been tested in technical feasibility studies $(24,25)$. The high temporal resolution allows a detailed morphological assessment of the uterus in numerous contrast phases at a spatial resolution higher than T2W BLADE and is even sufficient to generate parametric perfusion maps (25).

The goal of this study was to determine whether csVIBE in combination with T2W BLADE leads to an additional diagnostic benefit in terms of the detection rate of rectal infiltration, diagnostic confidence, and improved detection of the "mushroom cap sign," which is indicative of rectal infiltration.

\section{Patients and Methods}

Ethics statement. The study was approved by the ethics committee of our institution (2018-01586). The requirement for individual written informed consent was waived.

Patient selection and gold standard. The prototypical T1W gradientecho sequence (csVIBE) was employed in 151 women who underwent MRI examinations of the pelvis from May 24th 2018 to December $4^{\text {th }}, 2019$ (Figure 1). Among these, 80 MRI examinations performed to assess or exclude endometriosis were retrospectively selected from our imaging archive. The clinical information system was searched to determine which of these patients (age of $36 \pm 6$ years) had undergone a surgical procedure after the MRI examination by February 2020 at the latest $(n=43)$. From this group, the surgical reports were reviewed for patients with rectal wall infiltration due to DIE $(n=25)$. In two cases, rectal infiltration was surgically suspected but not confirmed histopathologically. The pathological report confirming the rectal infiltration by endometriosis was considered the gold standard for the study $(n=23)$. The cases in which the surgical report stated that a colorectal surgeon was consulted for possible rectosigmoid resection were classified as more extensive rectal invasion for statistical analysis $(n=13)$.

MRI acquisition. All examinations were performed with clinical 1.5T MRI machines (MAGNETOM Aera/Avantofit; Siemens Healthineers, Erlangen, Germany) equipped with a 50-channel coil setup (18channel body coil and 32-channel spine coil). The patients were positioned head-first in the supine position in the MRI machine. All patients abstained from food for 4 hours before the examination and were premedicated with glucagon to reduce peristalsis (26).

In our routine protocol, we do not use rectal opacification. The results in the literature are contradictory in this respect. While some authors postulate improved evaluation of the Douglas space and rectosigmoidal endometriosis (27-29), other studies have not shown a significant advantage in the detection of posterior DIE $(10,23)$.

csVIBE images were acquired during free respiration after administration of gadopentetate dimeglumine (Dotarem ${ }^{\circledR}$; Guerbet, Paris, France) as part of our clinical standard protocol and reconstructed immediately afterward using compressed sensing. For csVIBE, $0.1 \mathrm{mmol} / \mathrm{kg}$ of gadopentetate dimeglumine was administered at a rate of $2 \mathrm{ml} / \mathrm{s}$. Contrast was injected $5 \mathrm{~s}$ after the sequence had started. The temporal resolution ranged from $5 \mathrm{~s}$ in the initial phase to about $14 \mathrm{~s}$ in the later phases. The duration of the dynamic contrast-enhanced sequence was $2 \min 24 \mathrm{~s}$. The protocol parameters of T2W BLADE and csVIBE are detailed in Table I.

MRI assessment. Two radiologists with 20 years and 2 years of experience in female imaging (Reader A and B, respectively) separately performed a second reading of the MRI examinations of all operated patients $(n=43)$ without knowledge of the histopathological results.

Overall performance of T2W BLADE and T2W BLADE/csVIBE. First, only the sagittal T2W BLADE images were considered and evaluated with regard to the question of rectal infiltration. The sagittal contrast-enhanced dynamic T1W csVIBE images were subsequently included in the evaluation. When the two readers obtained different results $(n=5)$, a third reader with 10 years of experience in female imaging was consulted. The results of this consensus read were used for statistical evaluation.

Additional diagnostic value of csVIBE and scan preference. Both readers independently assessed the additional benefit of csVIBE in addition to T2W BLADE for the detection of rectal infiltration $(0=$ none, $1=$ moderate, $2=$ high $)$. Furthermore, the sequence preferred by both readers was noted $(0=$ equal, $1=\mathrm{csVIBE}$ preferred, 2=BLADE preferred)

Diagnostic confidence. Both the less experienced and the experienced reader rated the diagnostic confidence for the presence of rectal infiltration by DIE on T2W BLADE alone and on the combination of T2W BLADE and additional csVIBE $(0=$ low, $1=$ moderate, 2 =high). 


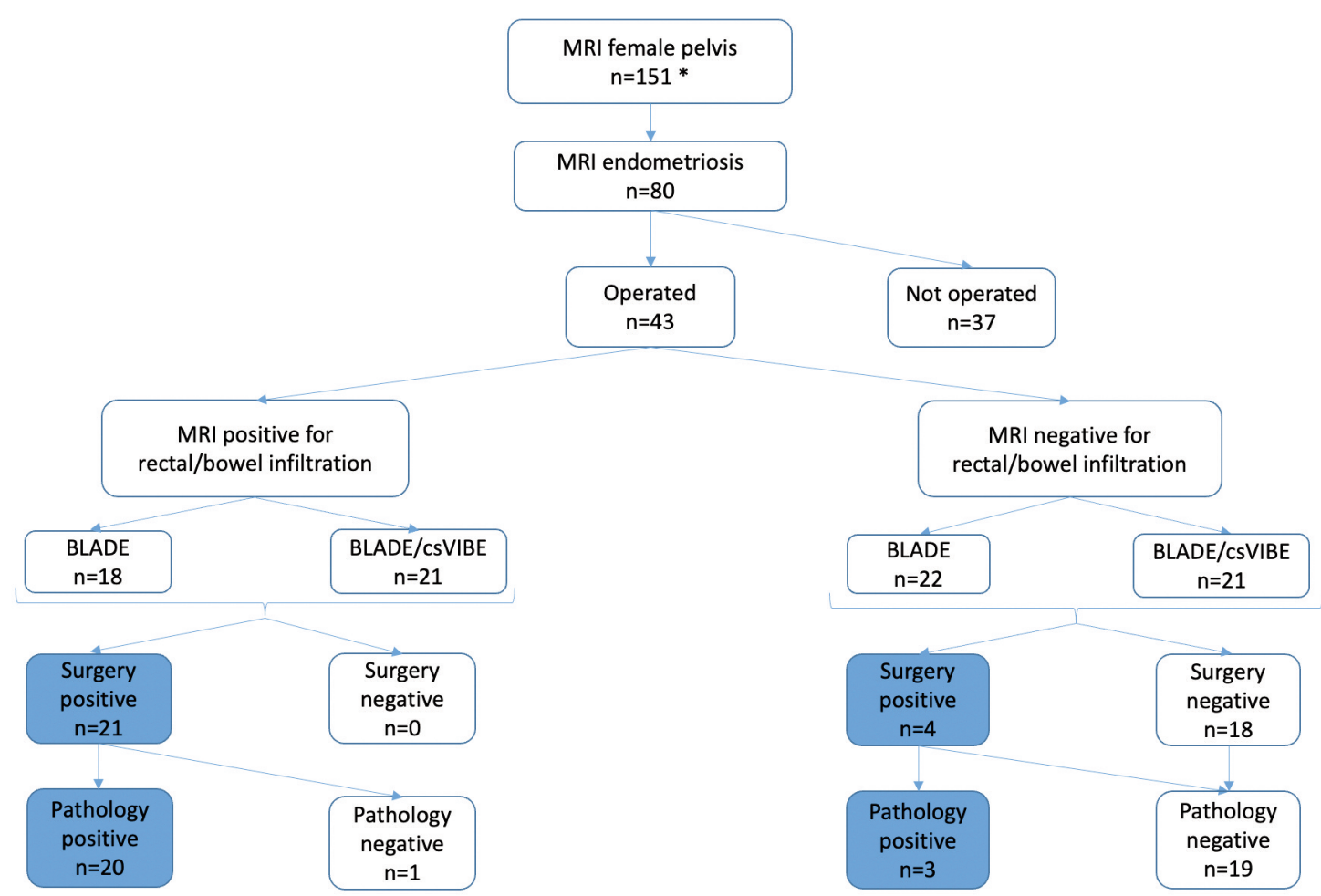

* First patient 24.05.2018, last patient 04.12.2019

Figure 1. Flowchart of study population. Of the 151 patients initially included, 43 underwent operations and were included in the study. Histopathological deep infiltrating endometriosis with rectal infiltration was confirmed in 23 cases. MRI: Magnetic resonance imaging; csVIBE: compressed-sensing volume-interpolated breath-hold examination.

\begin{abstract}
"Mushroom cap sign". The "mushroom cap sign" is a pathognomonic imaging sign that indicates the presence of extensive rectal infiltration and describes the characteristic appearance of the infiltration of endometriosis in the rectal wall (30, 31). Infiltration causes the mucosa to be raised, thus forming the roof of the fungus, and the extension to the rectum forms the stalk. Both readers assessed the presence of the mushroom cap sign ( $0=$ none, $1=$ unsure, $2=$ yes) first on T2W BLADE and then on csVIBE. Both readers' preferred sequence was determined.
\end{abstract}

Statistical analyses. All statistical analyses were performed using $\mathrm{R}$ version 3.6.3 (2020-02-29) and dedicated packages. All analyses were produced programmatically using the R Markdown format in RStudio, compliant with the principles of reproducible research (32). We determined whether the combined analysis of $\mathrm{T} 1 \mathrm{~W}$ and $\mathrm{T} 2 \mathrm{~W}$ images led to a more accurate diagnosis than analysis using T2W images alone, and we performed Fisher's exact test for each method and each reader. Accuracy, sensitivity, and specificity were calculated separately for each reader. We calculated the 95\% binomial proportion confidence interval and checked for significant differences in accuracy, sensitivity, and specificity between $\mathrm{T} 1 \mathrm{~W}+\mathrm{T} 2 \mathrm{~W}$ and $\mathrm{T} 2 \mathrm{~W}$ imaging alone with a twoproportion Z-test. Additionally, we investigated whether the combined analysis of $\mathrm{T} 1 \mathrm{~W}$ and $\mathrm{T} 2 \mathrm{~W}$ images improved the diagnostic confidence of rectal/bowel infiltration compared with
Table I. The most relevant sequence parameters of T2W BLADE and csVIBE.

\begin{tabular}{|c|c|c|c|c|c|c|}
\hline $\begin{array}{l}\text { Imaging } \\
\text { protocol }\end{array}$ & $\begin{array}{c}\text { Slice } \\
\text { thickness } \\
(\mathrm{mm})\end{array}$ & $\begin{array}{l}\text { FOV } \\
(\mathrm{mm})\end{array}$ & $\begin{array}{l}\text { TR } \\
(\mathrm{ms})\end{array}$ & $\begin{array}{l}\mathrm{TE} \\
(\mathrm{ms})\end{array}$ & $\begin{array}{c}\text { Flip angle } \\
\left({ }^{\circ}\right)\end{array}$ & $\begin{array}{l}\text { Temporal } \\
\text { resolution } \\
\text { (s) }\end{array}$ \\
\hline csVIBE & 2.5 & 260 & 4.75 & 1.83 & 12 & $4.6-13.8$ \\
\hline BLADE & 4.0 & 210 & 5730 & 88 & $\mathrm{n} / \mathrm{a}$ & $\mathrm{n} / \mathrm{a}$ \\
\hline
\end{tabular}

T2W: T2-weighted; csVIBE: compressed-sensing volume-interpolated breath-hold examination; FOV: field of view; TR: repetition time; TE: echo time; n/a: not available.

the analysis of $\mathrm{T} 2 \mathrm{~W}$ images alone, and we calculated the $95 \%$ Poisson confidence intervals for the observed counts of cases. In particular, we determined the odds ratio with the $95 \%$ confidence interval of increasing the confidence for $\mathrm{T} 1 \mathrm{~W}$ and $\mathrm{T} 2 \mathrm{~W}$ images compared with $\mathrm{T} 2 \mathrm{~W}$ images using ordinal regression. Finally, the preference of the mushroom subset was analyzed for both readers, and we used an exact Poisson test to identify any significant differences between the two methods. 
Table II. Confusion matrix of T2W BLADE and csVIBE for each rater separately.

\begin{tabular}{lccc}
\hline & No $(\mathrm{n}=19)$ & Yes $(\mathrm{n}=23)$ & Total $(\mathrm{n}=42)$ \\
\hline Age, years & & & \\
$\quad$ Mean \pm SD & $35.47 \pm 7.86$ & $36.26 \pm 5.10$ & $35.91 \pm 6.42$ \\
Range & $1-51$ & $28-45$ & $19-51$ \\
BLADE_A & & & \\
$\quad$ No & $18(94.7 \%)$ & $6(26.1 \%)$ & $24(57.1 \%)$ \\
Yes & $1(5.3 \%)$ & $17(73.9 \%)$ & $18(42.9 \%)$ \\
BLADE_B & & & \\
No & $18(94.7 \%)$ & $5(21.7 \%)$ & $23(54.8 \%)$ \\
Yes & $1(5.3 \%)$ & $18(78.3 \%)$ & $19(45.2 \%)$ \\
BLADE/csVIBE_A & & & \\
No & $15(78.9 \%)$ & $4(17.4 \%)$ & $19(45.2 \%)$ \\
Yes & $4(21.1 \%)$ & $19(82.6 \%)$ & $23(54.8 \%)$ \\
BLADE/csVIBE_B & & & \\
No & $18(94.7 \%)$ & $3(13.0 \%)$ & $21(50.0 \%)$ \\
Yes & $1(5.3 \%)$ & $20(87.0 \%)$ & $21(50.0 \%)$ \\
BLADE_Agree & & & \\
$\quad$ No & $18(94.7 \%)$ & $5(22.7 \%)$ & $23(56.1 \%)$ \\
$\quad$ Yes & $1(5.3 \%)$ & $17(77.3 \%)$ & $18(43.9 \%)$ \\
BLADE/csVIBE_Agree & $15(93.8 \%)$ & $3(13.6 \%)$ & $21(47.4 \%)$ \\
$\quad$ No & $1(6.2 \%)$ & $19(86.4 \%)$ & $21(52.6 \%)$ \\
Yes & & & \\
\hline
\end{tabular}

Note: $p$-Values (Fisher's exact test) were calculated for each confusion matrix. T2W: T2-weighted; csVIBE: compressed-sensing volumeinterpolated breath-hold examination; SD: standard deviation.

\section{Results}

Overall performance of T2W BLADE and T2W $B L A D E / c s V I B E$. The sensitivity, specificity, and accuracy of the consensus read for the assessment of rectal invasion was $0.77,0.95$, and 0.85 for sagittal T2W BLADE alone and $0.86,0.94$, and 0.90 for the combination of T2W BLADE and csVIBE (Tables II and III, Figure 2). Overall, the specificity was higher than the sensitivity, but there was no significant difference between the methods and readers. The mutual agreement rate between the readers was $97.62 \%$ for T2W BLADE with a Cohen's kappa of $0.95\left(p=6.61 \times 10^{-10}\right)$ and $90.48 \%$ for csVIBE with a Cohen's kappa of 0.81 $\left(p=1.36 \times 10^{-7}\right)$.

Additional diagnostic value of csVIBE and scan preference. Both readers preferred T2W BLADE over csVIBE (Reader A: $68.29 \%$, Reader B $63.41 \%$ ). CsVIBE added diagnostic value in $53.66 \%$ of the patients for Reader A and $56.10 \%$ for Reader B.

Diagnostic confidence. We investigated whether the combined analysis of csVIBE and T2W BLADE images improved the diagnostic confidence of rectal/bowel infiltration compared with analysis of T2W BLADE alone (Figure 3). The odds ratio of increasing the confidence for
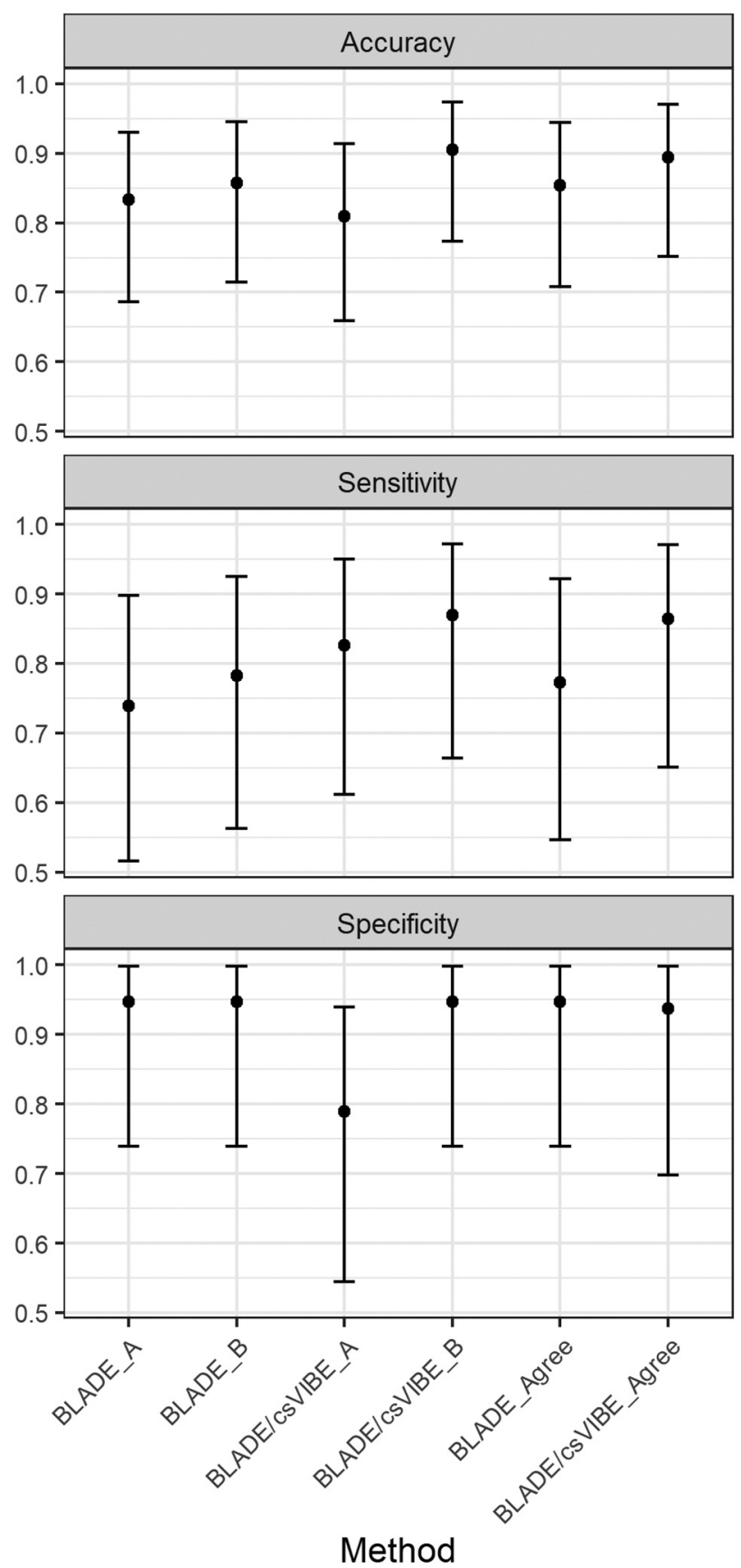

Figure 2. Accuracy, sensitivity, and specificity of the two methods (BLADE and csVIBE) and readers (Readers $A$ and B). csVIBE: Compressed-sensing volume-interpolated breath-hold examination.

T2W BLADE/csVIBE compared with T2W BLADE alone using ordinal regression for Reader A was 1.254 (2.5\%: $0.569,97.5 \%: 2.777)$, and that for Reader B was 1.520 (2.5\%: $0.684,97.5 \%$ : 3.420). For both readers, the confidence in the diagnosis increased with the addition of 
Table III. Accuracy, sensitivity, and specificity of the two methods.

\begin{tabular}{|c|c|c|c|c|c|c|}
\hline & Accuracy & $p$-Value & Specificity & $p$-Value & Sensitivity & $p$-Value \\
\hline BLADE_A & $0.833(0.686-0.93)$ & 1 & 0.947 (0.74-0.999) & 0.337 & $0.739(0.516-0.898)$ & 0.721 \\
\hline BLADE/csVIBE_A & $0.81(0.659-0.914)$ & & $0.789(0.544-0.939)$ & & $0.826(0.612-0.95)$ & \\
\hline BLADE_B & $0.857(0.715-0.946)$ & 0.736 & $0.947(0.74-0.999)$ & 1 & $0.783(0.563-0.925)$ & 0.697 \\
\hline BLADE/csVIBE_B & $0.905(0.774-0.973)$ & & $0.947(0.74-0.999)$ & & $0.87(0.664-0.972)$ & \\
\hline BLADE_Agree & $0.854(0.708-0.944)$ & 0.834 & 0.947 (0.74-0.999) & 1 & $0.773(0.546-0.922)$ & 0.696 \\
\hline BLADE/csVIBE_Agree & $0.895(0.752-0.971)$ & & $0.938(0.698-0.998)$ & & $0.864(0.651-0.971)$ & \\
\hline
\end{tabular}

Data are presented as the estimated value (95\% confidence interval). csVIBE: Compressed-sensing volume-interpolated breath-hold examination.
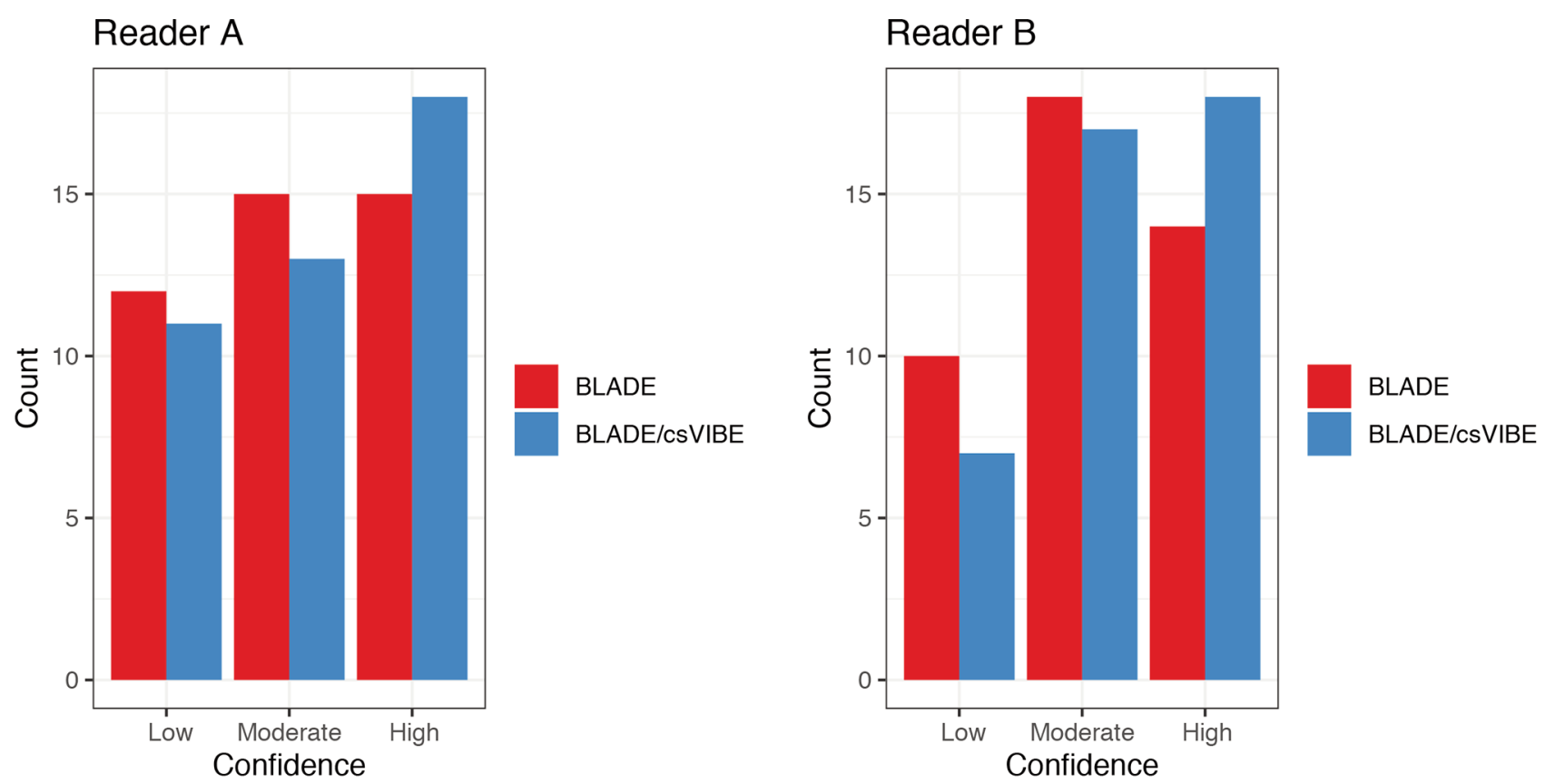

Figure 3. Raters' confidence in the methods. Red: BLADE, blue: BLADE/csVIBE. csVIBE: Compressed-sensing volume-interpolated breath-hold examination.

csVIBE, but this increase in the odds ratio was not statistically significant. We also compared the readers' confidence in the correctness of their prediction (Table IV). No misclassifications for predictions occurred, and confidence was high for both readers and methods. The predictions with moderate confidence were also correct in most cases. The number of incorrect predictions increased for predictions with low confidence.

"Mushroom cap sign". The "mushroom cap sign" was detected in nine patients. In this subset, T2W BLADE and csVIBE were directly compared regarding scan preference. csVIBE was preferred by both readers in the subset of the data with this imaging sign, with statistically significant differences (Reader A: $p=0.031$, Reader B: $p=0.031$ ) (Figures 4, 5, 6 and 7).

\section{Discussion}

In the present study, high diagnostic accuracy for the detection of rectal infiltration by endometriosis was determined with good to very good agreement between readers. The accuracy of csVIBE/T2W BLADE (90\%) was better than that of T2W BLADE alone $(85 \%)$. However, the differences were not significant for both readers and for the mutual agreement. This is explained by the fact that T2W BLADE already had very high accuracy and the overall sample size was too small to detect small differences. A power analysis was performed with the assumption that $\mathrm{T} 2 \mathrm{~W}$ BLADE had an accuracy of $80 \%$ and that the combination T2W BLADE/csVIBE had an accuracy of $90 \%$. To obtain an effect at a $5 \%$ significance level with a power of $80 \%$, the 
Table IV. Confusion matrix of raters' confidence.

\begin{tabular}{lccc}
\hline & FALSE $(\mathrm{n}=7)$ & TRUE $(\mathrm{n}=35)$ & Total $(\mathrm{n}=42)$ \\
\hline BLADE_A & & & \\
Low & $5(71.4 \%)$ & $7(20.0 \%)$ & $12(28.6 \%)$ \\
Moderate & $2(28.6 \%)$ & $13(37.1 \%)$ & $15(35.7 \%)$ \\
High & $0(0.0 \%)$ & $15(42.9 \%)$ & $15(35.7 \%)$ \\
FALSE (n=6) & TRUE $(\mathrm{n}=36)$ & Total $(\mathrm{n}=42)$ & \\
BLADE_B & & & \\
Low & $4(66.7 \%)$ & $6(16.7 \%)$ & $10(23.8 \%)$ \\
Moderate & $2(33.3 \%)$ & $16(44.4 \%)$ & $18(42.9 \%)$ \\
High & $0(0.0 \%)$ & $14(38.9 \%)$ & $14(33.3 \%)$ \\
FALSE (n=8) & TRUE $(\mathrm{n}=34)$ & Total $(\mathrm{n}=42)$ & \\
BLADE/csVIBE_A & & & \\
Low & $4(50.0 \%)$ & $7(20.6 \%)$ & $11(26.2 \%)$ \\
Moderate & $4(50.0 \%)$ & $9(26.5 \%)$ & $13(31.0 \%)$ \\
High & $0(0.0 \%)$ & $18(52.9 \%)$ & $18(42.9 \%)$ \\
FALSE (n=4) & TRUE $(\mathrm{n}=38)$ & Total $(\mathrm{n}=42)$ & \\
BLADE/csVIBE_B & & & \\
Low & $1(25.0 \%)$ & $6(15.8 \%)$ & $7(16.7 \%)$ \\
Moderate & $3(75.0 \%)$ & $14(36.8 \%)$ & $17(40.5 \%)$ \\
High & $0(0.0 \%)$ & $18(47.4 \%)$ & $18(42.9 \%)$ \\
\hline
\end{tabular}

csVIBE: Compressed-sensing volume-interpolated breath-hold examination.

optimal sample size would be 201 patients per group, which was not achievable.

The results of this study show that rectal infiltration can be clearly detected with a native protocol and that the administration of contrast medium only leads to relatively small diagnostic improvements. In individual cases $(n=3)$, however, rectal infiltration was only detectable after contrast agent administration.

In the literature, the sensitivity and specificity of MRI for bowel endometriosis ranges from $76 \%$ to $88 \%$ and from $76 \%$ to $80 \%$, respectively $(8,9,11,12)$. In the present study, sagittal T2W BLADE achieved a sensitivity of $77 \%$ and specificity of $95 \%$, while the combination of $\mathrm{T} 2 \mathrm{~W}$ BLADE/csVIBE achieved a sensitivity of $86 \%$ and specificity of $94 \%$ in identifying rectosigmoidal endometriosis.

In a retrospective evaluation of 158 patients by Bazot et al. (23), 3 readers achieved $77.2 \%, 73.4 \%$, and $86.1 \%$ accuracy, respectively, for the detection of rectosigmoid colon infiltration using a non-contrast protocol. The administration of contrast medium was of little additional value (accuracy of $77.8 \%, 83.5 \%$, and $87.3 \%$, respectively). There was no significant difference among the readers. As in our cohort, the diagnostic confidence could not be significantly improved by the administration of contrast medium $(p=0.67)(23)$.

Conversely, however, Scardapane et al. (22) detected a benefit from the administration of contrast medium. Among 104 patients, endometriosis was detected intraoperatively in 89 patients and DIE was diagnosed in 51 (57\%) of these 89 patients. Bowel endometriosis was further diagnosed in 21
$(41 \%)$ of the 51 patients. With the use of contrast-enhanced sequences, the sensitivity, specificity, positive predictive value, negative predictive value, and accuracy for the diagnosis of colorectal endometriosis improved from $76 \%$, $96 \%, 84 \%, 93 \%$, and $91 \%$ to $95 \%, 97 \%, 91 \%, 99 \%$ and 97 $\%$ for the experienced radiologist and from $62 \%, 93 \%, 72 \%$, $89 \%$, and $85 \%$ to $86 \%, 94 \%, 82 \%, 96 \%$, and $92 \%$ for the less experienced radiologist (22).

From a surgical point of view, reliable preoperative detection of rectosigmoid endometriosis and a detailed description of the depth of infiltration and the circumferential and craniocaudal extension is of decisive importance for the choice of surgical technique. Similar to MRI of the prostate and breast, structured reporting based on defined criteria is increasingly required, which demands high image quality (33). It is important to be able to preoperatively assess whether the operation can be performed by a gynecologic surgeon alone or whether a specialized rectal surgeon will have to be consulted because of the prospect of rectosigmoid resection. Rectosigmoid resection is associated with a risk of severe complications such as postoperative anastomotic leakage and rectovaginal fistula formation. In this regard, patients with extensive findings must be prospectively informed. Some authors even recommend a protective ileostomy for this reason.

Recent studies have shown that csVIBE, which combines high temporal and spatial resolution with excellent image quality through advanced artifact reduction despite sequence acquisition during free breathing, is superior to conventional contrast-enhanced sequences for both upper abdomen and female pelvic imaging $(24,25)$. Especially in the uterus, the different contrast phases produce good separation of the individual zones and allow for detailed assessment of the anatomy (25). In the present study, csVIBE enabled significantly improved recognition of the "mushroom cap sign," which indicates extensive rectal infiltration. These cases were often more serious, and a colorectal surgeon was required for the performance of rectosigmoid resection in the majority of operations (6 of 9 patients). csVIBE may therefore be important for better planning of the surgical procedure.

However, additional contrast-enhanced sequences are associated with increased time, resulting in higher examination costs. Because of the increase in the number of examinations and in order to save costs, efforts are now increasingly being made to perform the examination in a comprehensive manner. Therefore, based on our data, contrast-enhanced sequences for evaluation of endometriosis should be reserved for special cases. The current 2017 European Society of Urogenital Radiology guidelines also do not recommend contrast administration in the evaluation of DIE because of heterogeneous study results (5). There has been renewed discussion about the adverse effects of contrast 

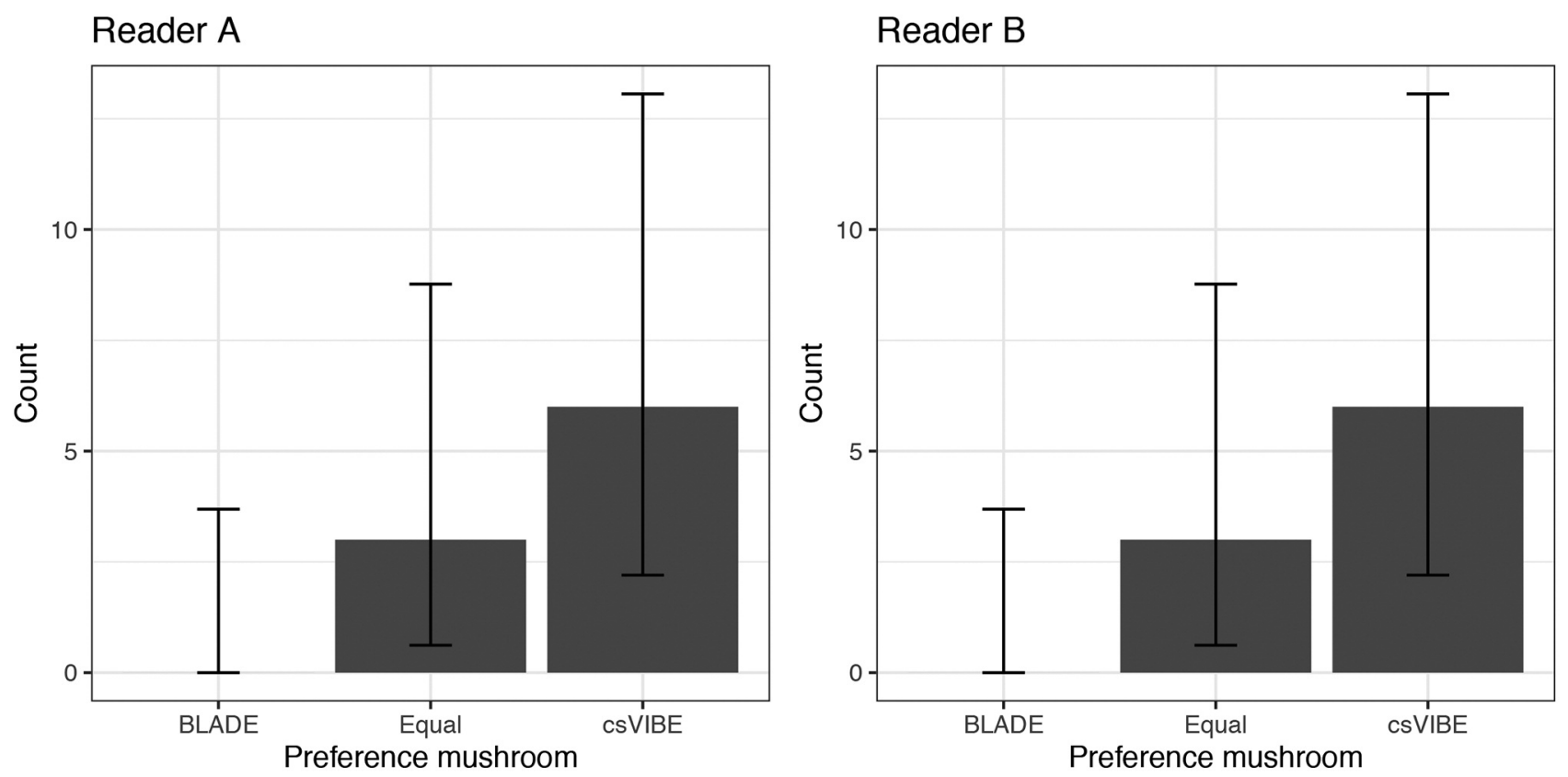

Figure 4. Sequence preference in the mushroom subset. csVIBE: Compressed-sensing volume-interpolated breath-hold examination.
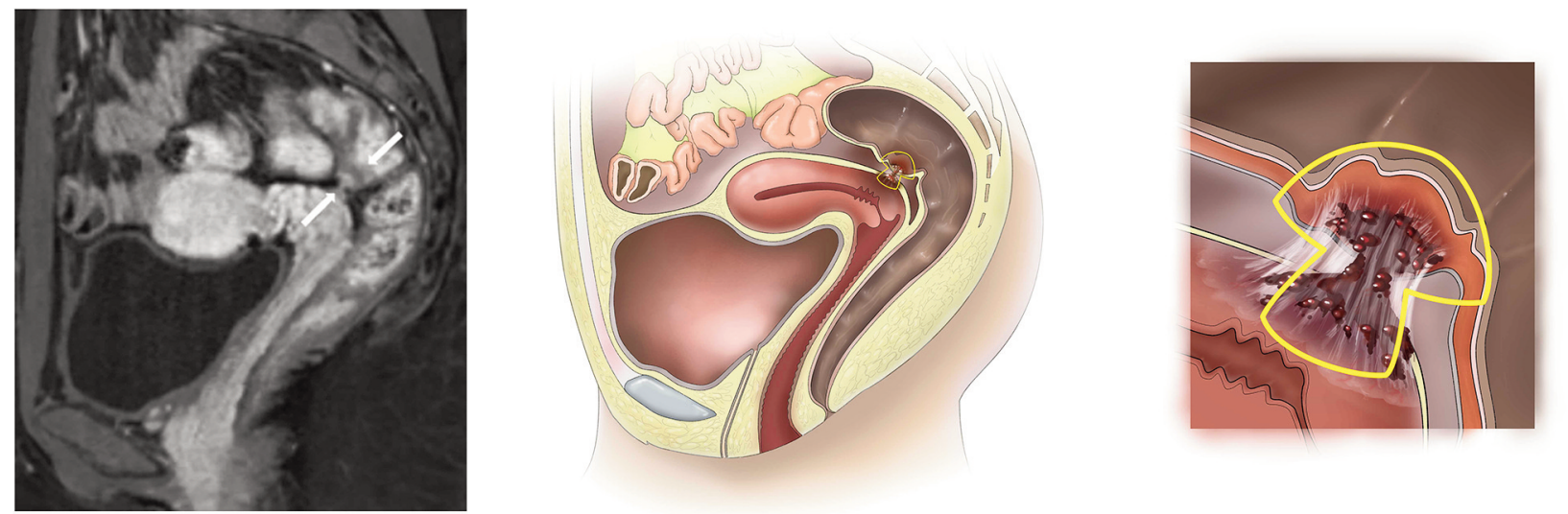

Figure 5. Images of a 22-year-old woman with deep infiltrating endometriosis and rectal involvement. The "mushroom cap sign" describes the appearance of rectal infiltration; the raised mucosa forms the roof of the fungus, while the extension to the rectum forms the stalk.

administration, such as cerebral gadolinium deposits of unclear significance and nephrogenic systemic fibrosis, especially with repeated contrast administration and in patients with impaired renal function (34-37).

Our study has some limitations. First, it was a retrospective evaluation. However, a prospective reevaluation was performed with the readers blinded to the histopathologic results. Second, the number of patients with DIE undergoing surgery was relatively small $(n=43)$. However, a power analysis showed that 201 patients per group would need to be included to obtain an effect at a 5\% significance level. This would not only be difficult to achieve but also underlines the fact that such a difference would probably not be of clinical significance in a routine setting. Finally, the results of our study are based on subjective assessments by the readers. No objective data were collected.

In accordance with the literature, MRI showed very good overall performance for the detection of rectosigmoidal endometriosis in the present study. The advantages of the addition of a contrast-enhanced dynamic sequence (T2W BLADE/csVIBE) compared with T2W imaging (BLADE) alone were not significant. Improved recognition of the 

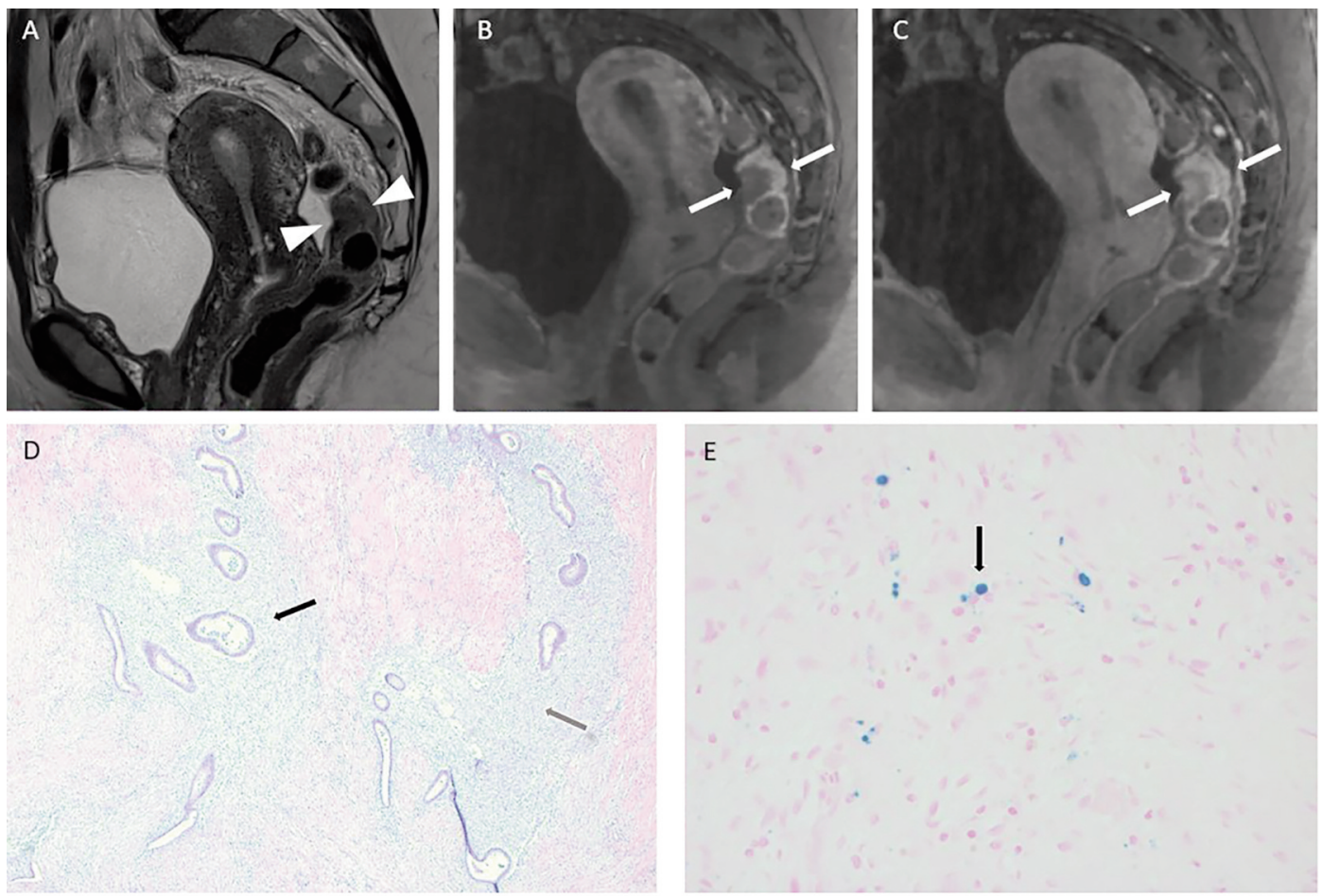

Figure 6. Images of a 32-year-old woman with deep infiltrating endometriosis. (A) The rectal infiltration was already clearly visible on T2-weighted BLADE. $(B, C)$ The "mushroom cap sign" was particularly clearly visible on csVIBE in different contrast phases. The sequence was preferred by both readers, and the additional diagnostic value was considered high. Histopathologic examination revealed (D) bland-appearing endometrial glands (black arrow) with associated dense stroma (grey arrow) between smooth muscle fibers of the intestine corresponding to deep infiltrating endometriosis and $(E)$ histiocytes with iron ingestion (iron stain, black arrow).
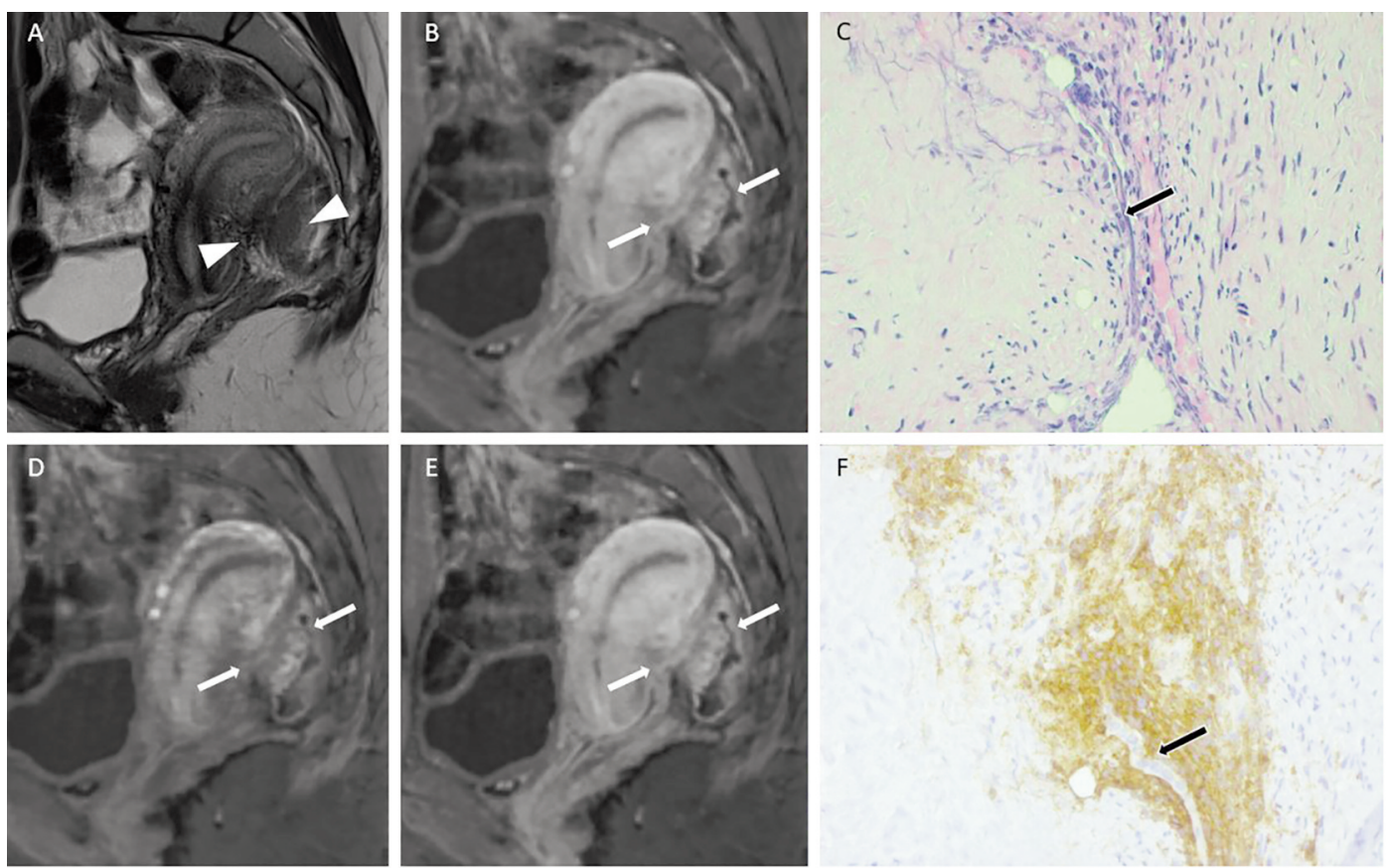

Figure 7. Images of another 32-year-old woman with extensive rectal endometriosis. Not (A) sagittal BLADE but (B-E) csVIBE was preferred by both readers. (C) Focal endometriosis (black arrow) was observed in connective tissue with fewer endometrial glands and scant stroma. (F) Endometrial stroma was immunohistochemically highlighted with an anti-CD10 antibody (neprilysin, brown stain), whereas glands did not stain (black arrow). 
"mushroom cap sign" is one reason why both readers found csVIBE to be of additional value. Because this sign indicates significant bowel involvement of DIE, which often leads to extensive surgery requiring the assistance of a colorectal surgeon, this could lead to an improvement in surgical planning in selected cases.

The question of whether the relatively small additional information gain from csVIBE justifies intravenous administration of contrast media, which is associated with increased costs and extension of the duration of the examination and thus a reduction in cost efficiency, must rather be negated.

\section{Conflicts of Interest}

The Authors declare that there are no conflicts of interest that could be perceived as prejudicing the impartiality of this study.

\section{Authors' Contributions}

All the Authors made substantial contributions to the conception and design, and/or acquisition of data, and/or analysis and interpretation of data. Also, all the Authors participated in drafting the article or gave final approval of the version to be submitted.

\section{Acknowledgements}

The Authors would like to thank Silke Callies (Department of Radiology, Kantonsspital Baden), Dr. M. Klarhöfer (Siemens Healthcare), Piotr Golofit (Radiologist, Medical Illustrator, Pomeranian Medical University, Szczecin, Poland), and Prof. G. Singer (Department of Pathology, Kantonsspital Baden) for their support in conducting this study and preparing the manuscript. The Authors also thank Angela Morben, DVM, ELS, from Edanz Group (https://en-authorservices.edanz.com/ac), for editing a draft of this manuscript.

\section{References}

1 Parasar P, Ozcan P and Terry KL: Endometriosis: epidemiology, diagnosis and clinical management. Curr Obstet Gynecol Rep 6(1): 34-41, 2017. PMID: 29276652. DOI: 10.1007/s13669-017$0187-1$

2 Dessy LA, Buccheri EM, Chiummariello S, Gagliardi DN and Onesti MG: Umbilical endometriosis, our experience. In Vivo 22(6): 811-815, 2008. PMID: 19181012.

3 Koninckx PR, Oosterlynck D, D'Hooghe T and Meuleman C: Deeply infiltrating endometriosis is a disease whereas mild endometriosis could be considered a non-disease. Ann N Y Acad Sci 734: 333-341, 1994. PMID: 7978935. DOI: 10.1111/j.17496632.1994.tb21763.x

4 Signorile PG, Campioni M, Vincenzi B, D'Avino A and Baldi A: Rectovaginal septum endometriosis: an immunohistochemical analysis of 62 cases. In Vivo 23(3): 459-464, 2009. PMID: 19454514.

5 Bazot M, Bharwani N, Huchon C, Kinkel K, Cunha TM, Guerra A, Manganaro L, Buñesch L, Kido A, Togashi K, ThomassinNaggara I and Rockall AG: European society of urogenital radiology (ESUR) guidelines: MR imaging of pelvic endometriosis. Eur Radiol 27(7): 2765-2775, 2017. PMID: 27921160. DOI: $10.1007 / \mathrm{s} 00330-016-4673-\mathrm{z}$

6 Anglesio MS and Yong PJ: Endometriosis-associated ovarian cancers. Clin Obstet Gynecol 60(4): 711-727, 2017. PMID: 28990985. DOI: $10.1097 /$ GRF.0000000000000320

7 Carter JE: Combined hysteroscopic and laparoscopic findings in patients with chronic pelvic pain. J Am Assoc Gynecol Laparosc 2(1): 43-47, 1994. PMID: 9050532. DOI: 10.1016/s10743804(05)80830-8

8 Abrao MS, Gonçalves MO, Dias JA Jr, Podgaec S, Chamie LP and Blasbalg R: Comparison between clinical examination, transvaginal sonography and magnetic resonance imaging for the diagnosis of deep endometriosis. Hum Reprod 22(12): 30923097, 2007. PMID: 17947378. DOI: 10.1093/humrep/dem 187

9 Bazot M, Darai E, Hourani R, Thomassin I, Cortez A, Uzan S and Buy JN: Deep pelvic endometriosis: MR imaging for diagnosis and prediction of extension of disease. Radiology 232(2): 379389, 2004. PMID: 15205479. DOI: 10.1148/radiol.2322030762

10 Hottat N, Larrousse C, Anaf V, Noël JC, Matos C, Absil J and Metens T: Endometriosis: contribution of 3.0-T pelvic MR imaging in preoperative assessment-initial results. Radiology 253(1): 126-134, 2009. PMID: 19584256. DOI: 10.1148/radiol. 2531082113

11 Bazot M, Bornier C, Dubernard G, Roseau G, Cortez A and Daraï E: Accuracy of magnetic resonance imaging and rectal endoscopic sonography for the prediction of location of deep pelvic endometriosis. Hum Reprod 22(5): 1457-1463, 2007. PMID: 17303630. DOI: 10.1093/humrep/dem008

12 Chapron C, Vieira M, Chopin N, Balleyguier C, Barakat H, Dumontier I, Roseau G, Fauconnier A, Foulot H and Dousset B: Accuracy of rectal endoscopic ultrasonography and magnetic resonance imaging in the diagnosis of rectal involvement for patients presenting with deeply infiltrating endometriosis. Ultrasound Obstet Gynecol 24(2): 175-179, 2004. PMID: 15287056. DOI: 10.1002/uog.1107

13 Moura APC, Ribeiro HSAA, Bernardo WM, Simões R, Torres US, D'Ippolito G, Bazot M and Ribeiro PAAG: Accuracy of transvaginal sonography versus magnetic resonance imaging in the diagnosis of rectosigmoid endometriosis: Systematic review and meta-analysis. PLoS One 14(4): e0214842, 2019. PMID: 30964888. DOI: 10.1371/journal.pone.0214842

14 Manganaro L, Fierro F, Tomei A, Irimia D, Lodise P, Sergi ME, Vinci V, Sollazzo P, Porpora MG, Delfini R, Vittori G and Marini M: Feasibility of $3.0 \mathrm{~T}$ pelvic MR imaging in the evaluation of endometriosis. Eur J Radiol 81(6): 1381-1387, 2012. PMID: 21497034. DOI: 10.1016/j.ejrad.2011.03.049

15 Di Paola V, Manfredi R, Castelli F, Negrelli R, Mehrabi S and Pozzi Mucelli R: Detection and localization of deep endometriosis by means of MRI and correlation with the ENZIAN score. Eur J Radiol 84(4): 568-574, 2015. PMID: 25604906. DOI: $10.1016 /$ j.ejrad.2014.12.017

16 Chamié LP, Blasbalg R, Gonçalves MO, Carvalho FM, Abrão MS and de Oliveira IS: Accuracy of magnetic resonance imaging for diagnosis and preoperative assessment of deeply infiltrating endometriosis. Int J Gynaecol Obstet 106(3): 198-201, 2009. PMID: 19467541. DOI: 10.1016/j.ijgo.2009.04.013

17 Dunselman GA, Vermeulen N, Becker C, Calhaz-Jorge C, D'Hooghe T, De Bie B, Heikinheimo O, Horne AW, Kiesel L, Nap A, Prentice A, Saridogan E, Soriano D, Nelen W and European Society of Human Reproduction and Embryology: 
ESHRE guideline: management of women with endometriosis Hum Reprod 29(3): 400-412, 2014. PMID: 24435778. DOI: 10.1093/humrep/det457

18 Togashi K, Nishimura K, Kimura I, Tsuda Y, Yamashita K, Shibata T, Nakano Y, Konishi J, Konishi I and Mori T: Endometrial cysts: diagnosis with MR imaging. Radiology 180(1): 73-78, 1991. PMID: 2052726. DOI: 10.1148/radiology. 180.1.2052726

19 Cornfeld DM, Israel G, McCarthy SM and Weinreb JC: Pelvic imaging using a $\mathrm{T} 1 \mathrm{~W}$ fat-suppressed three-dimensional dual echo Dixon technique at 3T. J Magn Reson Imaging 28(1): 121127, 2008. PMID: 18581401. DOI: 10.1002/jmri.21402

20 Forstner R, Thomassin-Naggara I, Cunha TM, Kinkel K, Masselli G, Kubik-Huch R, Spencer JA and Rockall A: ESUR recommendations for MR imaging of the sonographically indeterminate adnexal mass: an update. Eur Radiol 27(6): 2248-2257, 2017. PMID: 27770228. DOI: 10.1007/s00330016-4600-3

21 Onbas O, Kantarci M, Alper F, Kumtepe Y, Durur I, Ingec M, Gursan $\mathrm{N}$ and Okur A: Nodular endometriosis: dynamic MR imaging. Abdom Imaging 32(4): 451-456, 2007. PMID: 17420957. DOI: $10.1007 / \mathrm{s} 00261-006-9038-7$

22 Scardapane A, Bettocchi S, Lorusso F, Stabile Ianora AA, Vimercati A, Ceci O, Lasciarrea M and Angelelli G: Diagnosis of colorectal endometriosis: contribution of contrast enhanced MR-colonography. Eur Radiol 21(7): 1553-1563, 2011. PMID: 21336537. DOI: 10.1007/s00330-011-2079-5

23 Bazot M, Gasner A, Lafont C, Ballester M and Daraï E: Deep pelvic endometriosis: limited additional diagnostic value of postcontrast in comparison with conventional MR images. Eur J Radiol 80(3): e331-e339, 2011. PMID: 21216125. DOI: 10.1016/j.ejrad.2010.12.006

24 Hausmann D, Niemann T, Kreul D, Nocito A, Klarhöfer M, Nickel DM, Kiefer B, Attenberger UI, Zöllner FG and KubikHuch RA: Free-breathing dynamic contrast-enhanced imaging of the upper abdomen using a Cartesian compressed-sensing sequence with hard-gated and motion-state-resolved reconstruction. Invest Radiol 54(11): 728-736, 2019. PMID: 31503080. DOI: 10.1097/RLI.0000000000000607

25 Hausmann D, Kreul D, Klarhöfer M, Nickel D, Grimm R, Kiefer B, Riffel P, Attenberger UI, Zöllner FG and Kubik-Huch RA: Morphological and functional assessment of the uterus: "onestop shop imaging" using a compressed-sensing accelerated, free-breathing T1-VIBE sequence. Acta Radiol 62(5): 695-704, 2021. PMID: 32600068 . DOI: 10.1177/0284185120936260

26 Sala E, Rockall A and Kubik-Huch RA: Advances in magnetic resonance imaging of endometrial cancer. Eur Radiol 21(3): 468473, 2011. PMID: 21113597. DOI: 10.1007/s00330-010-2010-5

27 Faccioli N, Foti G, Manfredi R, Mainardi P, Spoto E, Ruffo G, Minelli L and Mucelli RP: Evaluation of colonic involvement in endometriosis: double-contrast barium enema $v s$. magnetic resonance imaging. Abdom Imaging 35(4): 414-421, 2010. PMID: 19568808. DOI: 10.1007/s00261-009-9544-5
28 Takeuchi H, Kuwatsuru R, Kitade M, Sakurai A, Kikuchi I, Shimanuki $\mathrm{H}$ and Kinoshita $\mathrm{K}$ : A novel technique using magnetic resonance imaging jelly for evaluation of rectovaginal endometriosis. Fertil Steril 83(2): 442-447, 2005. PMID: 15705388. DOI: 10.1016/j.fertnstert.2004.08.021

29 Kikuchi I, Kuwatsuru R, Yamazaki K, Kumakiri J, Aoki Y and Takeda S: Evaluation of the usefulness of the MRI jelly method for diagnosing complete cul-de-sac obliteration. Biomed Res Int 2014: 437962, 2014. PMID: 24812616. DOI: 10.1155/2014/437962

30 Arévalo N and Méndez R: "Mushroom cap" sign in deep rectosigmoid endometriosis. Abdom Radiol (NY) 43(11): 32013203, 2018. PMID: 29619523. DOI: 10.1007/s00261-018-1596-y

31 Yoon JH, Choi D, Jang KT, Kim CK, Kim H, Lee SJ, Chun HK, Lee WY and Yun SH: Deep rectosigmoid endometriosis: "mushroom cap" sign on T2-weighted MR imaging. Abdom Imaging 35(6): 726-731, 2010. PMID: 20820774. DOI: 10.1007/ s00261-010-9643-3

32 Peng RD: Reproducible research and biostatistics. Biostatistics 10(3): 405-408, 2009. PMID: 19535325. DOI: 10.1093/ biostatistics/kxp014

33 Mattos LA, Goncalves MO, Andres MP, Young SW, Feldman M, Abrão MS and Kho RM: Structured ultrasound and magnetic resonance imaging reports for patients with suspected endometriosis: guide for imagers and clinicians. J Minim Invasive Gynecol 26(6): 1016-1025, 2019. PMID: 30849475. DOI: $10.1016 /$ j.jmig.2019.02.017

34 Malikova H: Nephrogenic systemic fibrosis: the end of the story? Quant Imaging Med Surg 9(8): 1470-1474, 2019. PMID: 31559176. DOI: 10.21037/qims.2019.07.11

35 McDonald JS and McDonald RJ: $\mathrm{mr}$ imaging safety considerations of gadolinium-based contrast agents: Gadolinium retention and nephrogenic systemic fibrosis. Magn Reson Imaging Clin N Am 28(4): 497-507, 2020. PMID: 33040991. DOI: $10.1016 /$ j.mric.2020.06.001

36 Fingerhut S, Sperling M, Holling M, Niederstadt T, Allkemper T, Radbruch A, Heindel W, Paulus W, Jeibmann A and Karst U: Gadolinium-based contrast agents induce gadolinium deposits in cerebral vessel walls, while the neuropil is not affected: an autopsy study. Acta Neuropathol 136(1): 127-138, 2018. PMID: 29748901. DOI: 10.1007/s00401-018-1857-4

37 Radbruch A, Weberling LD, Kieslich PJ, Hepp J, Kickingereder P, Wick W, Schlemmer HP and Bendszus M: High-signal intensity in the dentate nucleus and globus pallidus on unenhanced T1-weighted images: Evaluation of the macrocyclic gadolinium-based contrast agent gadobutrol. Invest Radiol 50(12): 805-810, 2015. PMID: 26523910. DOI: 10.1097/ RLI.0000000000000227 